\title{
Unsteady Adaptive Stochastic Finite Elements for Aeroelastic Systems with Randomness
}

\author{
Jeroen A.S. Witteveen* and Hester Bijl ${ }^{\dagger}$ \\ Faculty of Aerospace Engineering, Delft University of Technology, The Netherlands
}

\begin{abstract}
It is recognized in the engineering community that there is an increasing need to move towards unsteady simulations in computational fluid dynamics. This trend also dictates an increasing application of uncertainty quantification methods to unsteady problems. In this paper, an Unsteady Adaptive Stochastic Finite Elements method based on interpolation at constant phase (UASFE-cp) is introduced for resolving the effect of random parameters in unsteady simulations. It achieves a constant accuracy in time with a constant number of samples, in contrast with the usually fast increasing number of samples required by other non-intrusive methods. Results for the stochastic bifurcation behavior of an elastically mounted airfoil with nonlinearity in the flow and the structure are presented.
\end{abstract}

\section{Introduction}

Most uncertainty quantification methods are mainly developed for steady problems such as steady heat transfer prediction. ${ }^{21}$ One of the most widely used uncertainty quantification methods is Monte Carlo simulation, ${ }^{8}$ in which many deterministic problems are solved for randomly varying parameter values. (Nonintrusive) Polynomial Chaos methods ${ }^{7,9,22}$ attempt to reduce the number of deterministic solves by using a polynomial interpolation of the samples in parameter space. An effective sampling in suitable Gauss quadrature points is employed in the Probabilistic Collocation approach. ${ }^{1}$ A more robust approximation is achieved by Stochastic Finite Elements methods, ${ }^{5,11,18}$ in which a piecewise polynomial interpolation of the samples is employed.

In unsteady problems non-intrusive uncertainty quantification methods usually require a fast increasing number of samples with time to maintain a constant accuracy. This behavior is caused by the increasing nonlinearity of the response surface for increasing integration times. This effect is especially profound in problems with oscillatory solutions in which the frequency of the response is affected by the random parameters. ${ }^{14}$ The frequency differences between the realizations lead to increasing phase differences with time, which in turn result in an increasingly oscillatory response surface and more samples. Asymptotic behavior is of practical interest in, for example, post-flutter analysis of fluid-structure interaction systems. ${ }^{15}$ Resolving the effect of input randomness in these long time integration problems requires a large number of deterministic computations. Especially in computationally intensive unsteady flow computations and fluid-structure interaction simulations, such a large sample size can lead to impractically high computational costs. For applications involving oscillatory motion a Fourier chaos basis has been developed by Millman et. al. ${ }^{12}$ Other unsteady applications can also be found in literature. ${ }^{2,13}$ In this paper, an efficient alternative approach for uncertainty quantification in oscillatory problems is proposed.

The concept of applying uncertainty quantification to a time-independent parametrization of unsteady samples instead of to the samples themselves was introduced by the authors to achieve a constant accuracy in time with a constant number of samples. ${ }^{16,20}$ The parametrization consist of a damping factor, frequency, relative phase, amplitude, a reference value, and the normalized (higher-period) shape function. Due to this time-independent parametrization the interpolation accuracy is independent of time. The time-independent parameterization was combined with a global polynomial Probabilistic Collocation ${ }^{1}$ interpolation in Probabilistic Collocation for Limit Cycle Oscillations (PCLCO). ${ }^{20}$ A more robust approximation was developed by

*PhD. student, j.a.s.witteveen@tudelft.nl, Kluyverweg 1, 2629HS Delft, AIAA Member.

${ }^{\dagger}$ Full Professor, h.bijl@tudelft.nl, Kluyverweg 1, 2629HS Delft. 
using an Adaptive Stochastic Finite Elements (ASFE) interpolation based on Newton-Cotes quadrature in simplex elements ${ }^{18}$ in the Unsteady Adaptive Stochastic Finite Elements method based on time-independent parametrization (UASFE-ti). ${ }^{17,19}$ It was demonstrated for, for example, an elastically mounted cylinder problem that the constant number of samples due to the time-independent parameterization results for long time integration problems in a reduction of computational costs by orders of magnitude compared to other methods. ${ }^{19,20}$

In this paper, the idea of performing the uncertainty quantification interpolation at constant phase is presented to further improve the accuracy and extend the applicability compared to time-independent parameterization, while maintaining a constant accuracy in time with a constant number of samples. As mentioned above, the usual increase of the number of samples with time is caused by increasing phase differences between the realizations. Scaling the oscillatory samples with their phase and performing the uncertainty quantification interpolation of the samples at constant phase instead of at constant time, eliminates the effect of the phase differences. The increase of the number of samples with time due to an increasingly oscillatory response surface is, therefore, avoided by interpolation at constant phase. In addition to the constant number of samples in time, interpolation at constant phase has the following three advantages over time-independent parametrization: (1) the parametrization error is eliminated; (2) time-dependent functionals can be resolved; and (3) transient behavior can be captured.

The uncertainty quantification interpolation at constant phase is performed using the Adaptive Stochastic Finite Elements (ASFE) method with Newton-Cotes quadrature and simplex elements. ${ }^{18}$ The resulting Unsteady Adaptive Stochastic Finite Elements method based on interpolation at constant phase (UASFEcp) can be applied to problems in which the phase of the oscillatory samples is well-defined. The UASFE-cp formulation is introduced in section II. The stochastic bifurcation behavior of the fluid-structure interaction system of nonlinear flow around an elastically mounted airfoil with nonlinear structural stiffness is analyzed in section III. Results for this application involving transient behavior in the post-bifurcation region are compared to those of UASFE-ti. The paper is concluded in section IV.

\section{Unsteady Adaptive Stochastic Finite Elements based on interpolation at constant phase}

The procedure for interpolation at constant phase in the Unsteady Adaptive Stochastic Finite Elements framework is developed in section II.A. The Adaptive Stochastic Finite Elements formulation employed for the interpolation is briefly reviewed in section II.B.

\section{II.A. Interpolation at constant phase}

Consider a dynamical system subject to $n$ uncorrelated second-order random input parameters $\mathbf{a}(\omega)=$ $\left\{a_{1}(\omega), \ldots, a_{n}(\omega)\right\} \in A$, which governs an oscillatory response $u(\mathbf{x}, t, \omega)$

$$
\mathcal{L}(\mathbf{x}, t ; u(\mathbf{x}, t, \omega))=S(\mathbf{x}, t)
$$

with operator $\mathcal{L}$ and source term $S$ defined on domain $D \times T$, and appropriate initial and boundary conditions. The spatial and temporal dimensions are defined as $\mathbf{x} \in D$ and $t \in T$, respectively, with $D \subset \mathbb{R}^{d}, d=\{1,2,3\}$, and $T=\left[0, t_{\text {max }}\right]$. A realization of the set of outcomes $\Omega$ of the probability space $(\Omega, \mathcal{F}, P)$ is denoted by $\omega \in \Omega$, with $\mathcal{F} \subset 2^{\Omega}$ the $\sigma$-algebra of events and $P$ a probability measure.

Assume that the phase of the oscillatory samples $u_{k}(t) \equiv u\left(t, \omega_{k}\right)$ for realizations of the random parameters $\mathbf{a}_{k} \equiv \mathbf{a}\left(\omega_{k}\right)$ is well defined for $k=1, \ldots, N_{\mathrm{s}}$. The argument $\mathbf{x}$ has been dropped here for convenience of the notation. In order to interpolate the samples $u_{k}(t)$ at constant phase, first, their phase as function of time $\phi_{k}(t)$ is extracted from the deterministic solves $u_{k}(t)$. Second, the time series for the phase $\phi_{k}(t)$ are used to transform the samples $u_{k}(t)$ to functions of their phase $u_{k}^{*}\left(\phi_{k}\right)$ instead of time. For discrete time histories the vectors $\vec{u}_{k}$ and $\vec{u}_{k}^{*}$ are identical. Third, the transformed samples $u_{k}^{*}\left(\phi_{k}\right)$ are interpolated to the function $u^{*}(\phi, \omega)$ using Adaptive Stochastic Finite Elements interpolation. This step involves both the interpolation of the sampled phases $\phi_{k}(t)$ to the function $\phi(t, \omega)$ and the interpolation of the samples $u_{k}^{*}(\tilde{\phi})$ to the function $u^{*}(\tilde{\phi}, \omega)$ at constant phase $\tilde{\phi}$. Repeating the latter interpolation for all phases $\tilde{\phi}$ results in the function $u^{*}(\phi, \omega)$. Finally, transforming $u^{*}(\phi, \omega)$ back to $u(t, \omega)$ using $\phi(t, \omega)$ yields the unknown response surface of the system response as function of the random parameters $\mathbf{a}(\omega)$ and time $t$. Integrating this response surface approximation results in an approximation of the statistical moments of the response. 
The phase $\phi_{k}(t)$ is extracted from the samples based on the local extrema of the time series $u_{k}(t)$. A trial and error procedure identifies a cycle of oscillation based on two or more successive local maxima. The selected cycle is accepted if the maximal error of its extrapolation in time with respect to the actual sample is smaller than a threshold value $\bar{\varepsilon}_{k}$ for at least one additional cycle length. The function for the phase $\phi_{k}(t)$ in the whole time domain $t \in T$ is constructed by identifying all successive cycles of $u_{k}(t)$ and extrapolation to $t=0$ and $t=t_{\max }$ before and after the first and last complete cycle, respectively. The phase is normalized to zero at the start of the first cycle and a user defined parameter determines whether the sample is assumed to attain a local extremum at $t=0$. If the phase $\phi_{k}(t)$ cannot be extracted from one of the samples $k=1, \ldots, N_{\mathrm{s}}$, Adaptive Stochastic Finite Elements interpolation can directly by applied to the time-dependent samples $u_{k}(t)$.

Since each sample reaches a different minimum and maximum phase in the time domain $t \in T$, the ASFE interpolation at constant phase is restricted to the range of phases that is reached by all samples in an element. The UASFE-cp interpolation is then limited to the time domain which corresponds to $\tilde{\phi} \in\left[\max _{k} \phi_{k}(0), \min _{k} \phi_{k}\left(t_{\max }\right)\right]$ in the elements. The time domain of approximation approaches $\left[0, t_{\max }\right]$ as the number of elements $N_{\mathrm{e}}$ increases. Outside this domain, ASFE can directly be applied to the timedependent samples $u_{k}(t)$.

\section{II.B. Adaptive Stochastic Finite Elements interpolation}

The interpolation of the samples at constant phase is performed in parameter space $A$ using a non-intrusive Adaptive Stochastic Finite Elements (ASFE) interpolation based on Newton-Cotes quadrature points in simplex elements. ${ }^{18}$ The ASFE formulation employs a piecewise quadratic approximation of the response surface by dividing parameter space $A$ into $N_{\mathrm{e}}$ simplex elements $A_{i}$ with $i=1, \ldots, N_{\mathrm{e}}$. The quadratic approximation in the elements is constructed by performing deterministic solves for the values of the random parameters $\mathbf{a}(\omega)$ that correspond to the $\left(\begin{array}{c}n+2 \\ 2\end{array}\right)$ second-degree Newton-Cotes quadrature points in the elements shown in Figure 1a. The two-dimensional example of Figure 1 can geometrically be extended to higherdimensional parameter spaces $A$.

The initial discretization of parameter space $A$ by the adaptive scheme consists of the minimum of $N_{\mathrm{e}_{\mathrm{ini}}}=n$ ! simplex elements and $N_{\mathrm{sini}}=3^{n}$ samples, see Figure 1b. The elements $A_{i}$ are adaptively refined as illustrated in Figure 1c using a refinement measure based on the largest absolute eigenvalue of the Hessian, as measure of the curvature of the response surface approximation in the elements, weighted by the probability represented by the elements. The actual refinement measure $\rho_{i}$ is the product of the refinement measures $\rho_{\phi_{i}}$ and $\rho_{\mathrm{u}_{i}^{*}}$ for the two ASFE interpolations of $\phi_{k}(t)$ to $\phi(t, \omega)$ and $u_{k}^{*}(\tilde{\phi})$ to $u^{*}(\tilde{\phi}, \omega)$, respectively. The stochastic grid refinement is terminated when $\delta_{N_{\mathrm{e}}}<\bar{\delta}$, where $\delta_{N_{\mathrm{e}}}$ is defined as

$$
\delta_{N_{\mathrm{e}}}=\max \left(\frac{\left|\mu_{\mathrm{u}_{\left\lfloor N_{\mathrm{e}} / 2\right\rfloor}}(t)-\mu_{\mathrm{u}_{N_{\mathrm{e}}}}(t)\right|_{\infty}}{\left|\mu_{\mathrm{u}_{N_{\mathrm{e}}}}(t)\right|_{\infty}}, \frac{\left|\sigma_{\mathrm{u}_{\left\lfloor N_{\mathrm{e}} / 2\right\rfloor}}(t)-\sigma_{\mathrm{u}_{N_{\mathrm{e}}}}(t)\right|_{\infty}}{\left|\sigma_{\mathrm{u}_{N_{\mathrm{e}}}}(t)\right|_{\infty}}\right),
$$

with $\mu_{\mathrm{u}}(t)$ and $\sigma_{\mathrm{u}}(t)$ the mean and standard deviation of $u(t, \omega)$, or when a threshold for the maximum number of samples $\bar{N}_{\mathrm{s}}$ is reached.

Due to the location of the Newton-Cotes quadrature points the required number of deterministic solves is relatively low, since the deterministic samples are reused in successive refinements and the samples are used in approximating the response in multiple elements. Where necessary the elements are subdivided into $N_{\mathrm{e}_{\text {lin }}}=$ $2^{n}$ subelements with a linear approximation of the response without performing additional deterministic solves to preserve monotonicity and extrema of the samples. This prevents unphysical predictions due to overshoots and undershoots near singularities at the expense that the method does not achieve exponential convergence for smooth responses as Galerkin and Gauss quadrature Stochastic Finite Elements methods can.

As is common in multi-element methods, the probability of the random parameters $\mathbf{a}(\omega)$ is assumed to be zero outside a finite domain. Probability distributions on infinite domains are truncated at a small enough threshold value for the probability, such that the truncation error is small compared to other numerical errors that occur in practical applications. 


\section{Stochastic bifurcation behavior of an elastically mounted airfoil}

A special class of responses that cannot be represented by time-independent parametrization consists of responses with a transient part. An application of practical interest with transient behavior is the (post)bifurcation analysis of an elastically mounted airfoil with nonlinear structural stiffness. The governing equations for a two-degree-of-freedom airfoil in an inviscid flow are outlined in section III.A. In section III.B the effect of randomness in the ratio of natural frequencies $\bar{\omega}(\omega)$ at the deterministic bifurcation point is studied. The P-bifurcation behavior of the probability distribution of the system response is analyzed in section III.C. The results are compared to those of UASFE-ti.

\section{III.A. Governing equations}

The nonlinear structural stiffness is modeled by a cubic spring stiffness term in the following two-degree-offreedom model for coupled pitch and plunge motion of the airfoil: ${ }^{6}$

$$
\begin{aligned}
& \xi^{\prime \prime}+x_{\alpha} \alpha^{\prime \prime}+\left(\frac{\bar{\omega}}{U^{*}}\right)^{2}\left(\xi+\beta_{\xi} \xi^{3}\right)=-\frac{1}{\pi \mu} C_{1}(\tau), \\
& \frac{x_{\alpha}}{r_{\alpha}^{2}} \xi^{\prime \prime}+\alpha^{\prime \prime}+\frac{1}{U^{* 2}}\left(\alpha+\beta_{\alpha} \alpha^{3}\right)=\frac{2}{\pi \mu r_{\alpha}^{2}} C_{\mathrm{m}}(\tau),
\end{aligned}
$$

where $\beta_{\xi}=0 \mathrm{~m}^{-2}$ and $\beta_{\alpha}=3 \mathrm{rad}^{-2}$ are nonlinear spring constants, $\xi(\tau)=h / b$ is the nondimensional plunge displacement of the elastic axis, see Figure 2a, $\alpha(\tau)$ is the pitch angle, and $\left(^{\prime}\right)$ denotes differentiation with respect to nondimensional time $\tau=U t / b$, with half-chord length $b=c / 2=0.5 \mathrm{~m}$ and free stream velocity $U=103.6 \mathrm{~m} / \mathrm{s}$, which corresponds to a Mach number of $M_{\infty}=0.3$ for free stream density $\rho_{\infty}=0.12 \mathrm{~kg} / \mathrm{m}^{3}$ and pressure $p_{\infty}=1.0 \cdot 10^{5} \mathrm{~Pa}$. The radius of gyration around the elastic axis is $r_{\alpha} b=0.25 \mathrm{~m}$, bifurcation parameter $U^{*}$ is defined as $U^{*}=U /\left(b \omega_{\alpha}\right)$, and the airfoil-air mass ratio is $\mu=m / \pi \rho_{\infty} b^{2}=100$, with $m$ the airfoil mass. The elastic axis is located at a distance $a_{\mathrm{h}} b=-0.25 \mathrm{~m}$ from the mid-chord position and the mass center is located at a distance $x_{\alpha} b=0.125 \mathrm{~m}$ from the elastic axis. ${ }^{10}$ Randomness is introduced in the ratio of natural frequencies $\bar{\omega}(\omega)=\omega_{\xi} / \omega_{\alpha}$, with $\omega_{\xi}$ and $\omega_{\alpha}$ the natural frequencies of the airfoil in pitch and plunge, respectively. The randomness is described by a symmetric unimodal beta distribution with parameters $\beta_{1}=\beta_{2}=2$ around a mean of $\mu_{\bar{\omega}}=0.2$ in the domain $\bar{\omega}(\omega) \in[0.15 ; 0.25]$.

The nondimensional aerodynamic lift and moment coefficients, $C_{\mathrm{l}}(\tau)$ and $C_{\mathrm{m}}(\tau)$, are determined by solving the nonlinear Euler equations for inviscid flow ${ }^{4}$ using a second-order finite volume scheme on an unstructured hexahedral mesh with $7.5 \cdot 10^{3}$ volumes in spatial domain $D$ with dimensions $30 c \times 20 c$. An Arbitrary Lagrangian-Eulerian formulation is employed to couple the fluid mesh with the movement of the structure. The fluid mesh is deformed using radial basis function interpolation of the boundary displacements. ${ }^{3}$ Time integration is performed using the BDF-2 method with stepsize $\Delta \tau=0.4$ until $\tau_{\max }=1000$. Initially the airfoil is at rest at a deflection of $\alpha(0)=0.1 \mathrm{deg}$ and $\xi(0)=0$ from its equilibrium position. The initial condition of the flow field is given by the steady state solution for the initial deflection shown in Figure $2 \mathrm{~b}$ in terms of the static pressure $p$. The output of interest is the angle of attack $\alpha(\tau, \omega)$. The plunge deflection $\xi(\tau, \omega)$ gives equivalent results.

In the deterministic case, the airfoil exhibits an oscillation which either decays to zero or diverges until it reaches a limit cycle oscillation after a transient, depending on the value of bifurcation parameter $U^{*}$. The deterministic bifurcation behavior of the system for the mean frequency ratio $\mu_{\bar{\omega}}$ is given in Figure 3a in terms of the amplitude $A_{\alpha}$ of pitch angle $\alpha(\tau)$ as function of normalized bifurcation parameter $U^{*} / U_{L}^{*}$. The linear flutter point $U_{L}^{*}$ is determined numerically by a trial and error procedure for the linear system with $\beta_{\alpha}=0$. The location of the flutter point $U^{*} / U_{L}^{*}=1$ is not affected by the nonlinear term, since the onset of flutter is a linear phenomenon. For $U^{*} / U_{L}^{*}=1$ the response is a linearly stable periodic motion of which the amplitude $A_{\alpha}$ is approximately equal to the initial condition $\alpha(0)$. The system shows a supercritical Hopf-bifurcation with zero amplitudes $A_{\alpha}$ below the flutter point for $U^{*} / U_{L}^{*}<1$ and finite amplitudes $A_{\alpha}$ increasing with $U^{*} / U_{L}^{*}$ for $U^{*} / U_{L}^{*}>1$. In the next section the effect of randomness in $\bar{\omega}(\omega)$ on the response at the deterministic bifurcation point $U^{*} / U_{L}^{*}=1$ is resolved. The stochastic system bifurcation as function of bifurcation parameter $U^{*} / U_{L}^{*}$ is analyzed in section III.C. 


\section{III.B. Transient behavior captured}

The large effect of randomness in the frequency ratio $\bar{\omega}(\omega)$ on the linearly stable response in the deterministic bifurcation point is illustrated by the $N_{\mathrm{sini}}=3$ initial samples for $\bar{\omega}_{k} \in\{0.15 ; 0.2 ; 0.25\}$ in Figure 3b. Sample $\alpha_{k}(\tau)$ for the mean value of the frequency ratio $\mu_{\bar{\omega}}=0.2$ is periodic with amplitude $A_{\alpha} \approx \alpha(0)$ as expected. Decreasing the frequency ratio to $\bar{\omega}=0.15$ results in a qualitative change to a damped response. On the other hand, increasing $\bar{\omega}$ to 0.25 leads to the onset of unstable behavior, which a single deterministic simulation for the mean value $\mu_{\bar{\omega}}$ would have missed. The corresponding realization shows a transient diverging oscillation until $\tau=400$ where it reaches a periodic limit cycle oscillation with a constant amplitude of $A_{\alpha}=8.0 \mathrm{deg}$ due the hard spring structural nonlinearity. The system, therefore, shows a supercritical Hopf bifurcation as function of $\bar{\omega}(\omega)$ as well.

The effect of the transient behavior of part of the realizations on the time histories of the mean $\mu_{\alpha}(\tau)$ and standard deviation $\sigma_{\alpha}(\tau)$ is shown in Figure 4 for UASFE-cp with $N_{\mathrm{e}}=5$ and $N_{\mathrm{e}}=10$ elements. The results are compared to those of UASFE-ti with $N_{\mathrm{e}}=10$. The mean $\mu_{\alpha}(\tau)$ is initially a diverging oscillation due to diverging transient oscillation of part of the realizations. For $\tau>600$ the mean shows a decaying oscillation due to the effect of $\bar{\omega}(\omega)$ on the frequency of the response in combination with the constant limit cycle oscillation amplitude of the post-flutter realizations beyond their transient. The transient contributes also to the fast initial increase of the standard deviation $\sigma_{\alpha}(\tau)$ to $2.3 \mathrm{deg}$ at $\tau \approx 1000$, which is more than a factor 20 larger than the deterministic amplitude $A_{\alpha} \approx 0.1 \mathrm{deg}$ of the periodic oscillation for $\mu_{\bar{\omega}}$. The successive UASFE-cp approximations for $N_{\mathrm{e}}=5$ and $N_{\mathrm{e}}=10$ elements display the convergence of the adaptive refinement. It has been verified that it requires $N_{\mathrm{e}}=16$ elements to obtain a comparable degree of convergence using uniform grid refinement. The adaptive refinement, therefore, results here in a $36 \%$ reduction of the required number of samples from $N_{\mathrm{s}}=33$ to $N_{\mathrm{s}}=21$. The UASFE-ti formulation does not predict the initial increase of $\mu_{\alpha}(\tau)$ and $\sigma_{\alpha}(\tau)$, since it is unable to model the transient behavior of the samples $\alpha_{k}(\tau)$. The large errors in the UASFE-ti approximation compared to the UASFE-cp results demonstrate the importance of capturing transient behavior.

\section{III.C. Stochastic bifurcation behavior}

By repeating the preceding analysis for a range of values of bifurcation parameter $U^{*} / U_{L}^{*}$, the stochastic bifurcation behavior of the system can be explored. Due to the random frequency ratio $\bar{\omega}(\omega)$, the system bifurcation can no longer be described by a single deterministic flutter point, but rather by a P-bifurcation region for the probability distribution. The response surface approximation of $\alpha(\omega)$ at $\tau=800$ as function of $\bar{\omega}(\omega)$ for $U^{*} / U_{L}^{*} \in[0.95 ; 1.05]$ is shown in Figure 5 . The bifurcation of the probability distribution as function of $U^{*} / U_{L}^{*}$ is here the primary output of interest. The two-dimensional surface of Figure 5 is, therefore, constructed using one-dimensional approximations as function of $\bar{\omega}(\omega)$ at discrete steps $\Delta U^{*} / U_{L}^{*}=0.0125$ given by the black lines instead of a full two-dimensional response surface approximation. A bifurcation line in the $\bar{\omega}-U^{*} / U_{L}^{*}$ plane can clearly be identified between $\left(\bar{\omega}, U^{*} / U_{L}^{*}\right)=(0.25 ; 0.975)$ and $\left(\bar{\omega}, U^{*} / U_{L}^{*}\right)=$ $(0.15 ; 1.025)$. For lower values of $\varpi(\omega)$ and $U^{*} / U_{L}^{*}$ the response is a pre-bifurcation decaying oscillation which results at $\tau=800$ in a pitch angle $\alpha(\omega)$ close to zero. The bifurcation region starts at $U^{*} / U_{L}^{*}=0.975$ for $\bar{\omega}=0.25$ and extends to $U^{*} / U_{L}^{*}=1.025$, after which the system develops a fully oscillatory response. In the post-bifurcation domain for $U^{*} / U_{L}^{*}>1.025$ the maximum deflection $|\alpha(\omega)|$ continues to increase with increasing $U^{*} / U_{L}^{*}$.

These results are reflected in the P-bifurcation behavior of the cumulative probability distribution function of $|\alpha(\omega)|$ at the discrete values of $U^{*} / U_{L}^{*}$ and $\tau=800$ in Figure 6. The cumulative distribution is shown here since it enables quantitative observations about the probability of flutter. The solutions based on two different grids with $N_{\mathrm{e}}$ and $\left\lfloor N_{\mathrm{e}} / 2\right\rfloor$ elements as used in convergence criterion (2) are shown to display the convergence of the approximations. In order to capture the stochastic bifurcation the required number of elements $N_{\mathrm{e}}$ is in the bifurcation region of $U^{*} / U_{L}^{*} \in[0.975 ; 1.025]$ up to 5 times larger than in the preor post-bifurcation domain. In the pre- as well as the post-bifurcation range a discretization with a single element is actually already sufficient.

When we define the bifurcation point as the absolute pitch angle $|\alpha(\omega)|$ which corresponds to a $50 \%$ probability of flutter at the deterministic flutter point $U^{*} / U_{L}^{*}=1$ in Figure $6 \mathrm{e}$, then we can determine the probability of flutter as function of bifurcation parameter $U^{*} / U_{L}^{*}$. The pre-bifurcation domain of $U^{*} / U_{L}^{*}<$ 0.975 shows a $100 \%$ probability of a damped response in Figures $6 \mathrm{a}$ and $6 \mathrm{~b}$. The $3.8 \%$ probability of postflutter behavior at $U^{*} / U_{L}^{*}=0.975$ indicates the start of the P-bifurcation. The probability of flutter increases 
further with increasing $U^{*} / U_{L}^{*}$ to $24.2 \%$ and $94.5 \%$ at $U^{*} / U_{L}^{*}=0.9875$ and $U^{*} / U_{L}^{*}=1.0125$, respectively. The maximal possible pitch angle $|\alpha(\omega)|$ continues to increase with $U^{*} / U_{L}^{*}$ in the post-bifurcation domain for $U^{*} / U_{L}^{*} \geq 1.025$, in which the probability of flutter is larger than $99.5 \%$. These results indicate that based on the assumed input probability distribution the acceptance of $24.2 \%, 3.8 \%$, or $0 \%$ probability of flutter would reduce the location of the stochastic bifurcation point by $1.25 \%, 2.5 \%$, or $3.75 \%$ compared to its deterministic position, respectively. The availability of this quantitative probabilistic information can be utilized in practice in reducing the safety factors in actual designs.

A typical P-bifurcation can also be recognized in the evolution of the probability density of amplitude $A_{\alpha}(\omega)$ for increasing $U^{*} / U_{L}^{*}$ at $\tau=800$ in Figure 7 . In the pre-bifurcation domain of $U^{*} / U_{L}^{*}<0.975$ the probability density resembles a Dirac delta function at $A_{\alpha}=0$. At $U^{*} / U_{L}^{*}=0.9875$ the probability still has a maximum at $A_{\alpha}=0$ and decays monotonically to zero for positive values of $A_{\alpha}$. The probability density develops a local maximum at a positive amplitude $A_{\alpha}=5.7 \mathrm{deg}$ for $U^{*} / U_{L}^{*}=1$. The local maximum increases and occurs at increasing values of $A_{\alpha}$ until it turns into the global most probable point at $U^{*} / U_{L}^{*}=1.025$. In the post-bifurcation domain of $U^{*} / U_{L}^{*} \geq 1.025$ the unimodal probability density function allows for positive $A_{\alpha}$ values only. The increased insight into the bifurcation behavior of the elastically mounted airfoil through these detailed stochastic results demonstrates the additional value of a stochastic analysis compared to a deterministic simulation for complex physical systems.

\section{Conclusions}

The concept of performing the uncertainty quantification interpolation at constant phase is presented to achieve a constant accuracy in time with a constant number of samples, while resolving time-dependent functionals such as in transient behavior. The usually fast increasing number of samples with time in other methods is caused by an increasingly oscillatory response surface with time due to increasing phase differences between the realizations. Scaling the oscillatory samples with their phase and performing the uncertainty quantification interpolation of the samples at constant phase instead of at constant time, eliminates this effect of the increasing phase differences. The interpolation at constant phase is performed using a robust Adaptive Stochastic Finite Elements (ASFE) method based on Newton-Cotes quadrature in simplex elements. The resulting Unsteady Adaptive Stochastic Finite Elements method based on interpolation at constant phase (UASFE-cf) can be applied to problems in which the phase of the oscillatory samples is well-defined.

Results for an elastically mounted airfoil with nonlinearity in the flow and the structure demonstrate that randomness in the frequency ratio $\bar{\omega}(\omega)$ can lead to the onset of unstable behavior, which a single deterministic simulation for mean value $\mu_{\bar{\omega}}$ would have missed. The UASFE-ti formulation does not capture the fast initial amplification of the standard deviation $\sigma_{\alpha}(\tau)$ of the pitch angle $\alpha(\omega)$ caused by the transient behavior of the samples to more than a 20 times its deterministic amplitude. The adaptive refinement of UASFE-cp results, for this problem, in a $36 \%$ reduction of the required number of samples to $N_{\mathrm{s}}=21$ compared to uniform grid refinement. The bifurcation of the cumulative probability distribution of the absolute pitch angle $|\alpha(\omega)|$ shows that due to the random $\bar{\omega}(\omega)$ a $2.5 \%$ reduction of the flutter boundary compared to its deterministic location still results in a $3.8 \%$ probability of flutter. A stochastic bifurcation analysis of the probability density of pitch amplitude $A_{\alpha}(\omega)$ reveals a typical P-bifurcation behavior from a Dirac delta function at zero amplitude to a unimodal probability density function for positive amplitudes.

\section{Acknowledgments}

This research was supported by the Technology Foundation STW, applied science division of NWO and the technology programme of the Ministry of Economic Affairs.

\section{References}

${ }^{1}$ I.M. Babuška, F. Nobile, R. Tempone, A stochastic collocation method for elliptic partial differential equations with random input data, SIAM J. Numer. Anal. 45(3) (2007) 1005-1034.

${ }^{2}$ P.S. Beran, C.L. Pettit, D.R. Millman, Uncertainty quantification of limit cycle oscillations, J. Comput. Phys. 217(1) (2006) 217-247.

${ }^{3}$ A. de Boer, M.S. van der Schoot, H. Bijl, Mesh deformation based on radial basis function interpolation, Comput. Struct. 85 (2007) 784-795.

${ }^{4}$ A.J. Chorin, J.E. Marsden, A mathematical introduction to fluid mechanics, Springer-Verlag, New York, 1979. 
${ }^{5}$ M.K. Deb, I.M. Babuška, J.T. Oden, Solution of stochastic partial differential equations using Galerkin finite element techniques, Comput. Methods Appl. Mech. Eng. 190 (2001) 6359-6372.

${ }^{6}$ Y. Fung, An introduction to aeroelasticity, Dover Publications, New York, 1969.

${ }^{7}$ R.G. Ghanem, P.D. Spanos, Stochastic finite elements: a spectral approach, Springer-Verlag, New York, 1991.

${ }^{8}$ J.M. Hammersley, D.C. Handscomb, Monte Carlo methods, Methuen's monographs on applied probability and statistics, Methuen, London, 1964.

${ }^{9}$ S. Hosder, R. W. Walters, R. Perez, A non-intrusive polynomial chaos method for uncertainty propagation in CFD simulations, AIAA-2006-891, 44 ${ }^{\text {th }}$ AIAA Aerospace Sciences Meeting and Exhibit, Reno, NV, 2006.

${ }^{10}$ B.H.K. Lee, L.Y. Jiang, Y.S. Wong, Flutter of an airfoil with a cubic nonlinear restoring force, $39^{\text {th }}$ AIAA/ASME/ASCE/AHS/ASC Structures, Structural Dynamics, and Materials Conference, Long Beach, CA (1998) AIAA1998-1725.

${ }^{11}$ O.P. Le Maître, H.N. Najm, R.G. Ghanem, O.M. Knio, Multi-resolution analysis of Wiener-type uncertainty propagation schemes, J. Comput. Phys. 197 (2004) 502-531.

${ }^{12}$ D.R.Millman, P.I. King, P.S. Beran, Airfoil pitch-and-plunge bifurcation behavior with Fourier chaos expansions, J. Aircraft 42(2) (2005) 376-384.

${ }^{13}$ C.L. Pettit, P.S. Beran, Effects of parametric uncertainty on airfoil limit cycle oscillation, J. Aircraft 40(5) (2003) 10041006.

${ }^{14}$ C.L. Pettit, P.S. Beran, Spectral and multiresolution Wiener expansions of oscillatory stochastic processes, J. Sound Vib. 294 (2006) 752-779.

${ }^{15}$ J.A.S. Witteveen, S. Sarkar, H. Bijl, Modeling physical uncertainties in dynamic stall induced fluid-structure interaction of turbine blades using arbitrary polynomial chaos, Comput. Struct. 85 (2007) 866-878.

${ }^{16}$ J.A.S. Witteveen, G.J.A. Loeven, H. Bijl, Quantifying the effect of physical uncertainties in unsteady fluid-structure interaction problems, AIAA-2007-1942, 48th AIAA/ASME/ASCE/AHS/ASC Structures, Structural Dynamics, and Materials Conference, Honolulu, HI, 2007.

${ }^{17}$ J.A.S. Witteveen, G.J.A. Loeven, H. Bijl, Long-term stochastic behavior of aeroelastic systems, NATO AVT-147 Conference on Computational Uncertainty in Military Vehicle Design, Athens, Greece, 2007.

18 J.A.S. Witteveen, G.J.A. Loeven, H. Bijl, An adaptive stochastic finite elements approach based on Newton-Cotes quadrature in simplex elements (2007) submitted.

${ }^{19}$ J.A.S. Witteveen, H. Bijl, An unsteady adaptive stochastic finite elements formulation for rigid-body fluid-structure interaction (2007) submitted.

${ }^{20}$ J.A.S. Witteveen, G.J.A. Loeven, S. Sarkar, H. Bijl, Probabilistic Collocation for period-1 limit cycle oscillations, J. Sound Vib. 311(1-2) (2008) 421-439.

${ }^{21}$ J.A.S. Witteveen, H. Bijl, Efficient quantification of the effect of uncertainties in advection-diffusion problems using polynomial chaos, Numer. Heat Tr. B-Fund. 53 (2008) 1-29.

22 J.A.S. Witteveen, H. Bijl, A monomial chaos approach for efficient uncertainty quantification in nonlinear problems, SIAM J. Sci. Comput. (2008) in press: doi:10.1137/06067287X

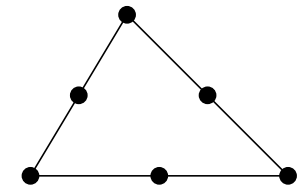

(a) Element

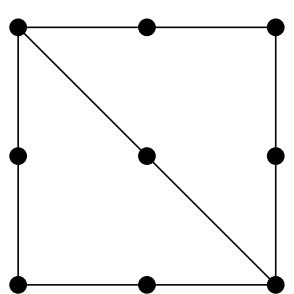

(b) Initial grid

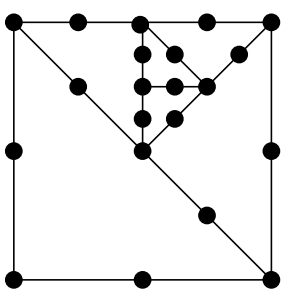

(c) Adapted grid

Figure 1. Discretization of two-dimensional parameter space $A$ using 2-simplex elements and second-degree NewtonCotes quadrature points given by the dots. 


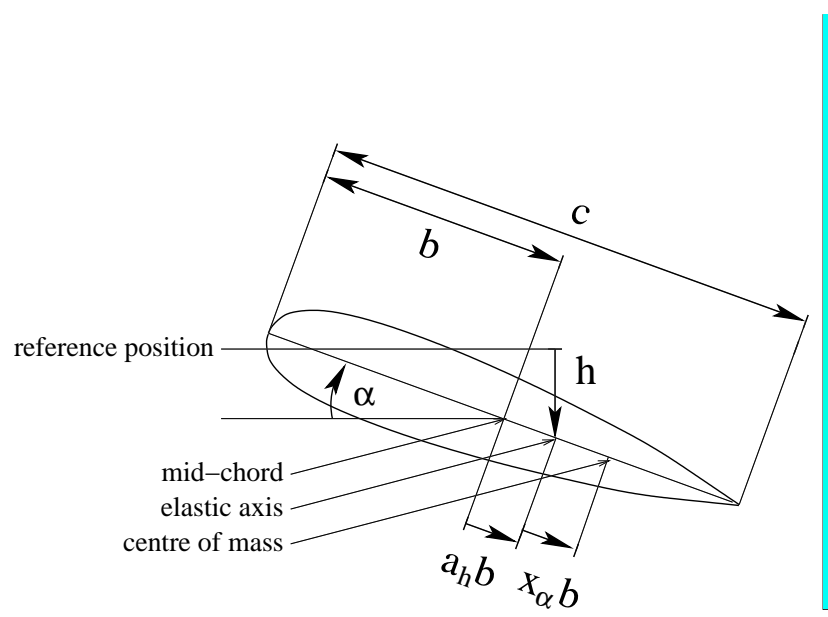

(a) structural model

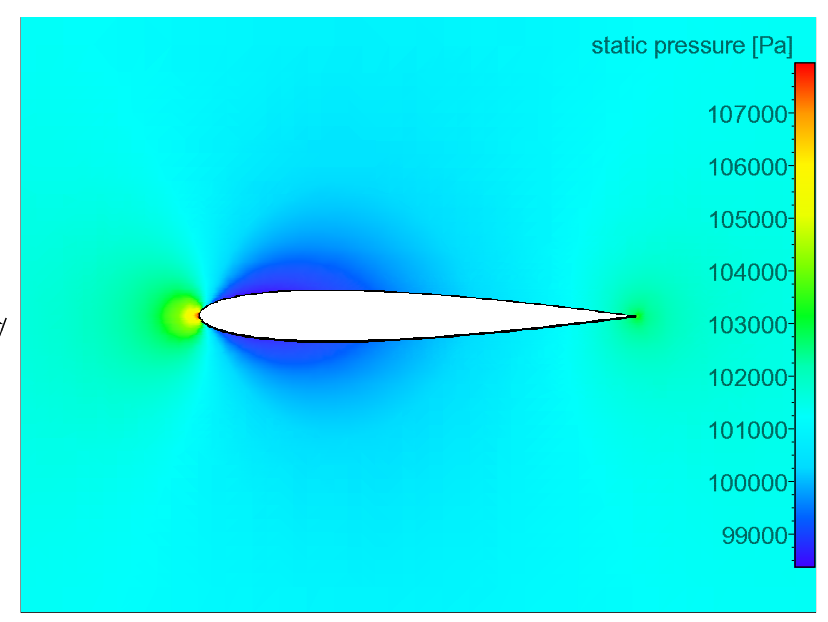

(b) initial flow conditions

Figure 2. The elastically mounted airfoil in uniform Euler flow.

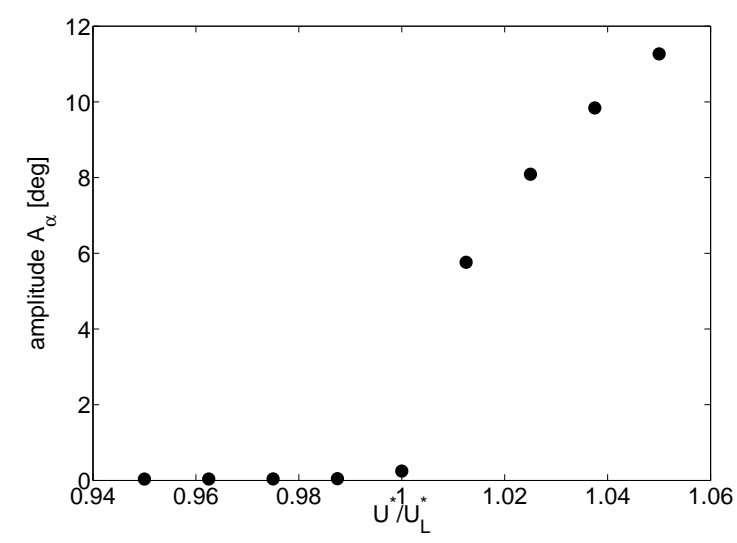

(a) deterministic bifurcation diagram

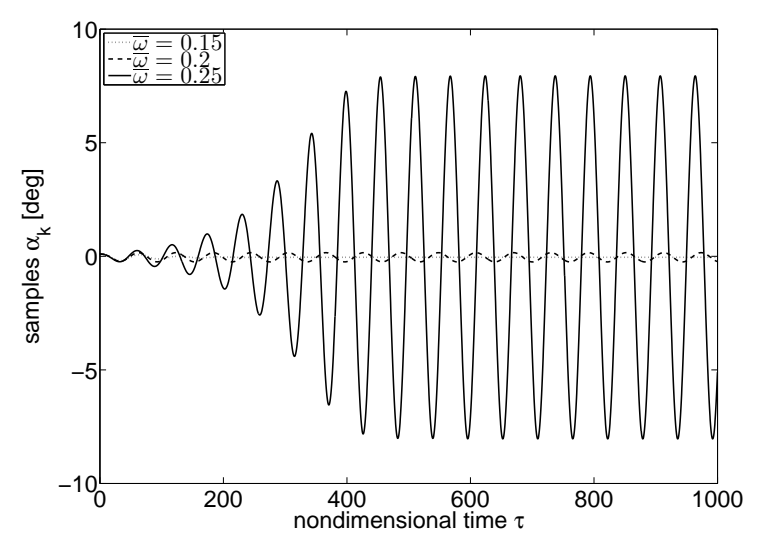

(b) initial $N_{\mathrm{s}_{\text {ini }}}=3$ UASFE-cp samples

Figure 3. Results for the elastically mounted airfoil.

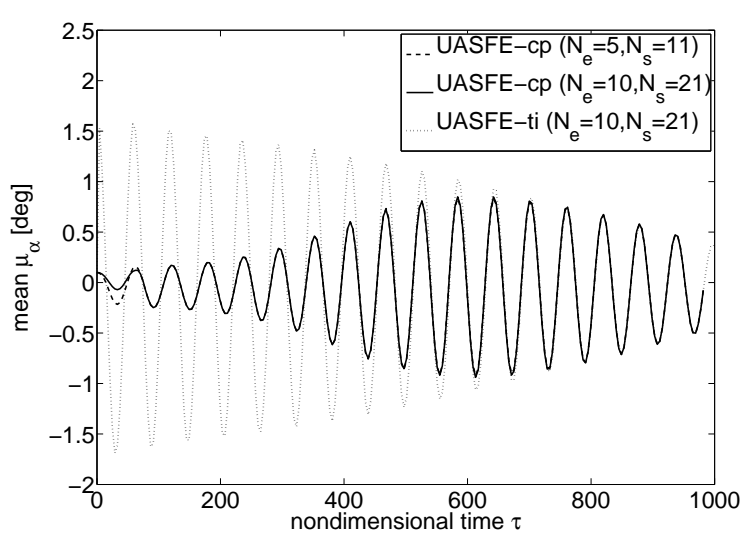

(a) mean $\mu_{\alpha}(\tau)$

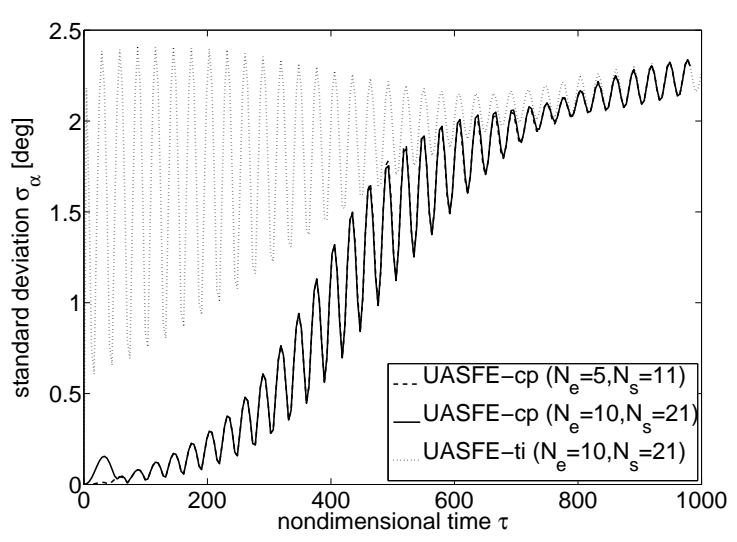

(b) standard deviation $\sigma_{\alpha}(\tau)$

Figure 4. Results of UASFE-cp with $N_{\mathrm{e}}=5$ elements and $N_{\mathrm{s}}=11$ samples, and $N_{\mathrm{e}}=10$ and $N_{\mathrm{s}}=21$ compared to those of UASFE-ti with $N_{\mathrm{e}}=10$ and $N_{\mathrm{s}}=21$ for the elastically mounted airfoil with $U^{*} / U_{L}^{*}=1$. 


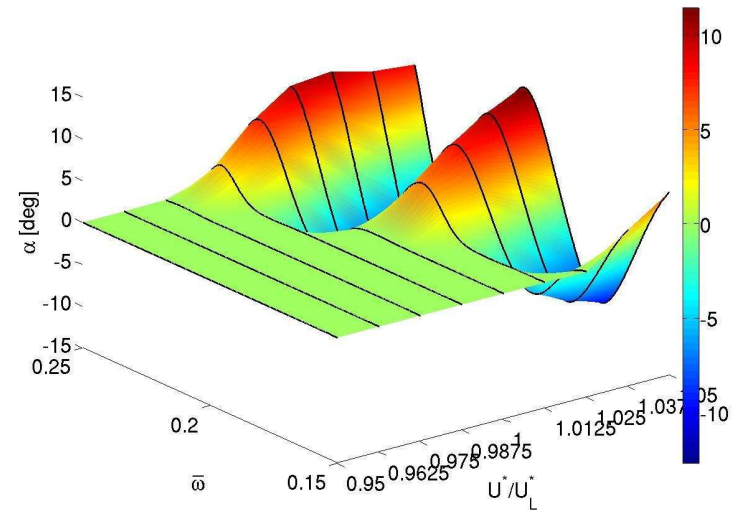

Figure 5. Response surface approximation of $\alpha(\omega)$ at $\tau=800$ as function of random $\bar{\omega}(\omega)$ and bifurcation parameter $U^{*} / U_{L}^{*}$ by UASFE-cp for the elastically mounted airfoil.

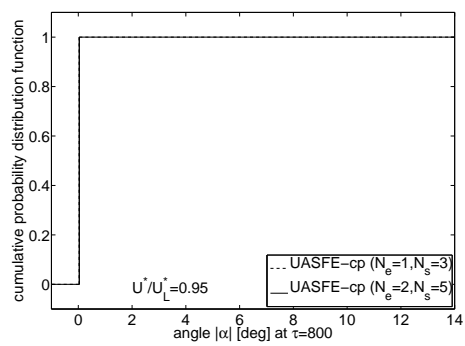

(a) $U^{*} / U_{L}^{*}=0.95$

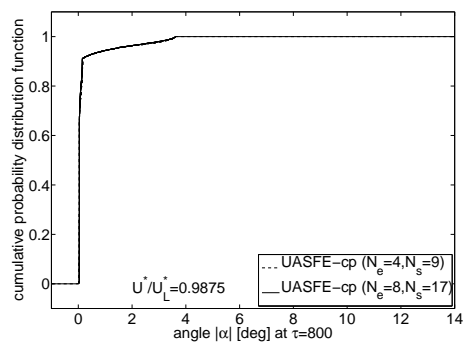

(d) $U^{*} / U_{L}^{*}=0.9875$

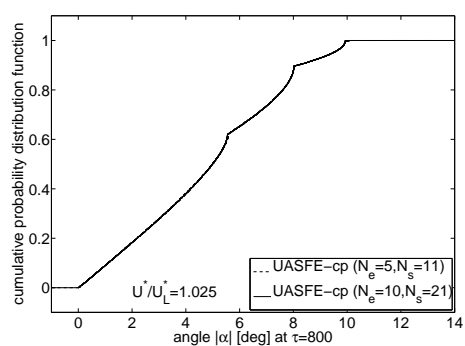

(g) $U^{*} / U_{L}^{*}=1.025$

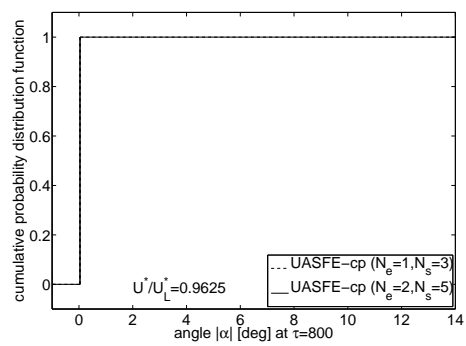

(b) $U^{*} / U_{L}^{*}=0.9625$

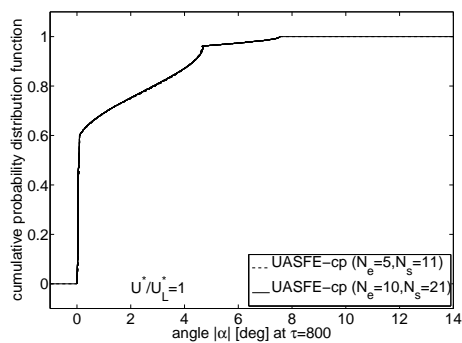

(e) $U^{*} / U_{L}^{*}=1$

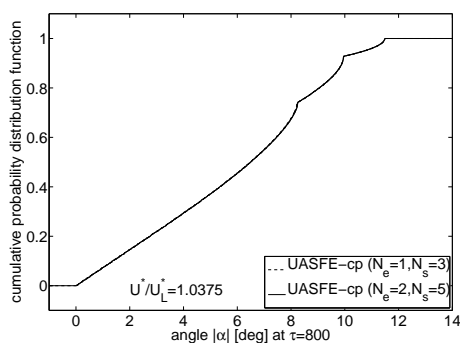

(h) $U^{*} / U_{L}^{*}=1.0375$

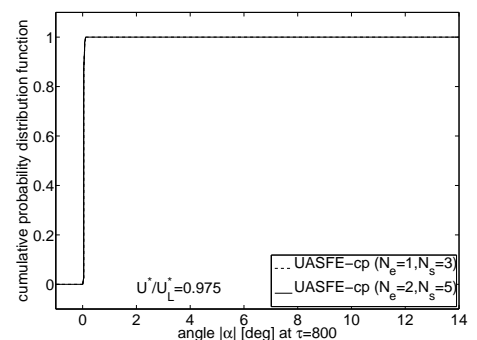

(c) $U^{*} / U_{L}^{*}=0.975$

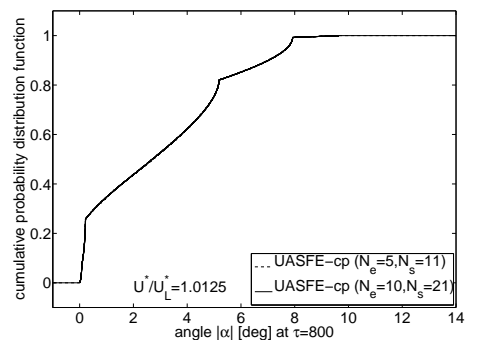

(f) $U^{*} / U_{L}^{*}=1.0125$

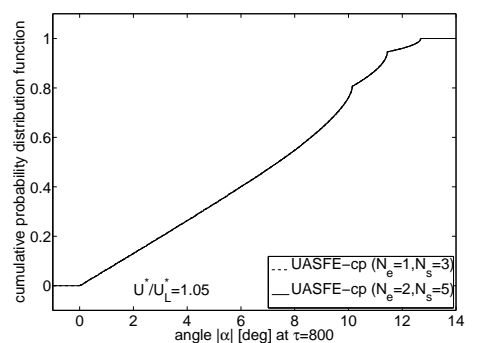

(i) $U^{*} / U_{L}^{*}=1.05$

Figure 6. Cumulative probability distribution of absolute pitch angle $|\alpha(\omega)|$ for $U^{*} / U_{L}^{*} \in[0.95 ; 1.05]$ at $\tau=800$ for the elastically mounted airfoil. 


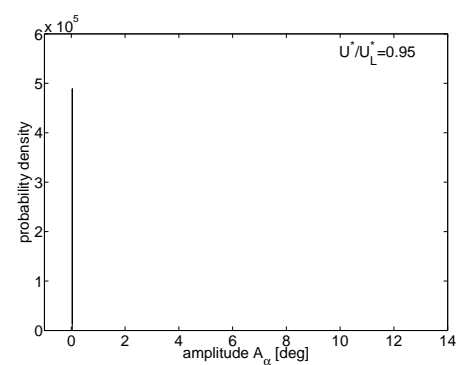

(a) $U^{*} / U_{L}^{*}=0.95$

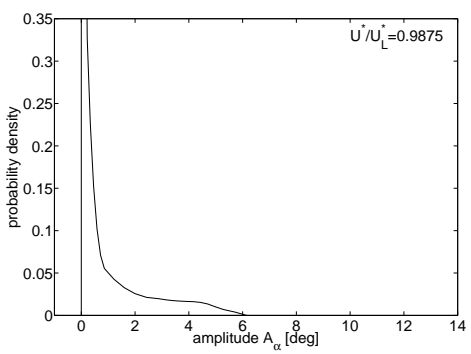

(d) $U^{*} / U_{L}^{*}=0.9875$

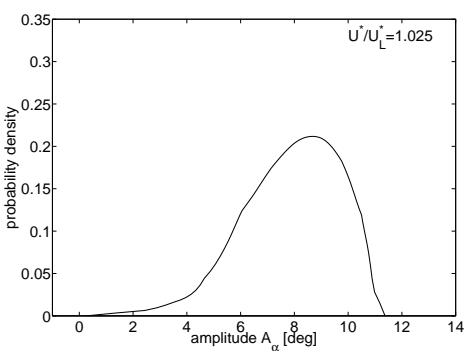

(g) $U^{*} / U_{L}^{*}=1.025$

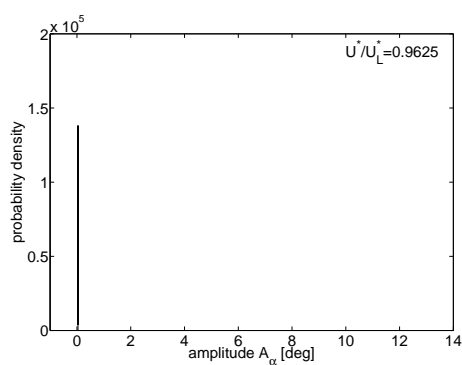

(b) $U^{*} / U_{L}^{*}=0.9625$

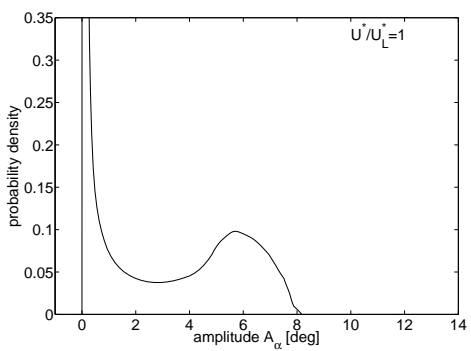

(e) $U^{*} / U_{L}^{*}=1$

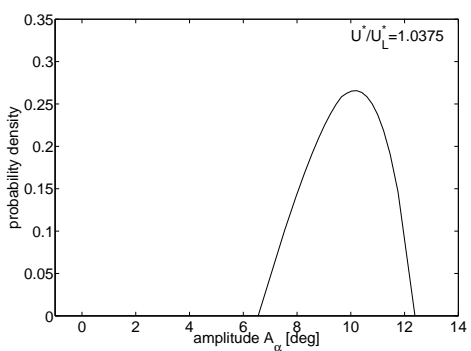

(h) $U^{*} / U_{L}^{*}=1.0375$

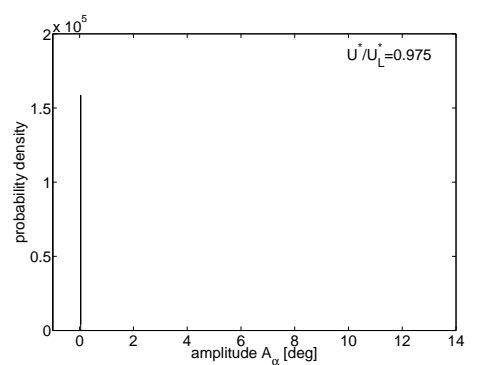

(c) $U^{*} / U_{L}^{*}=0.975$

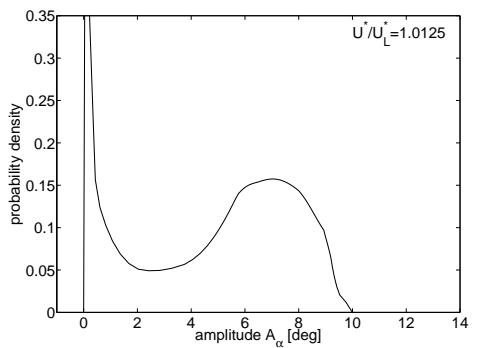

(f) $U^{*} / U_{L}^{*}=1.0125$

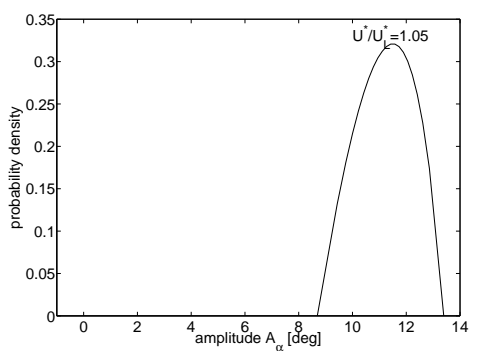

(i) $U^{*} / U_{L}^{*}=1.05$

Figure 7. Probability density of amplitude $A_{\alpha}(\omega)$ for $U^{*} / U_{L}^{*} \in[0.95 ; 1.05]$ at $\tau=800$ for the elastically mounted airfoil. 